UDK 821.133.1(493)-2.09Maeterlinck M.:27-23

\title{
L'HABITUS DE LA BIBLE DANS L' GUVRE DRAMATIQUE DE MAURICE MAETERLINCK
}

\author{
Boštjan Marko Turk
}

\section{Synopsis :}

L'étude présente se donne pour objectif d'établir le rapport entre l'univers de la Bible et l'œuvre dramatique de Maurice Maeterlinck. En fait, l'auteur belge vouait une prédilection aux sujets relatifs à l'Ancien et au Nouveau Testament. Ceux-ci mettent en relief l'intégralité de l'existence de l'homme, ce qui présente ipso facto un soubassement sans pareil pour l'exploatation dramatique, à tel point les histoires de la Bible sont imprégnées de dynamisme, reposant sur l'eschatologie du tragique et de l'inévitable. Maurice Maeterlinck en tant que dramaturge poursuit ces principes jusqu'aux moindres détails ce qui fait de lui un auteur important de l'inspiration biblique.

Mots clefs : Maurice Maeterlinck, Dieu, Bible, drame, existence, mort, résurrection, homme, tragique, miracle, mise en abyme

»Au fond, la poésie suprême n'est que cela, elle n'a d'autre but que de tenir ouvertes les grandes routes qui mènent à ce qu'on ne voit pas«. ${ }^{1}$

Maurice Maeterlinck reliait intimement les points de départs philosophiques avec les genres littéraires dans lesquels il écrivait. Son intérêt était tourné vers l'existence humaine, ou plus précisément, vers les possibilités spéculatives que les expériences ontologiques supérieures d'homo sapiens sapiens, ne soient pas reliées au support du cerveau, matière dans laquelle d'ailleurs ces expériences se produisent, et par conséquent sont indépendants de l'arrêt des fonctions organiques du corps appelé communément : la mort. Dans l'œuvre étendu et diversifié qu'il a laissé derrière lui, il est difficile de trouver un dénominateur commun autre que l'appréhension de la relation individualisée à l'extrême que chaque individu (de même que toute l'espèce en tant qu'entité globale) crée envers l'inévitable conclusion suivant chaque existence et par conséquent envers la question : qu'arrive-t-il après cela ? Le point de vue général de Maeterlinck serait alors que la vie est un phénomène universel se révélant aussi chez les autres êtres sous la forme qui est propre à l'homme. On ne peut comprendre les œuvres

\footnotetext{
${ }^{1}$ Claudia Chinatti. »Fausto Maria Martini, poète crépusculaire italien, disciple de Maeterlinck «. Annales, 11. Bruxelles : Maurice Maeterlinck Stichting, $1965: 22$.
} 
comme : L'Intelligence des Fleurs, ${ }^{2}$ La Vie des Abeilles, ${ }^{3}$ La Vie des Termites ${ }^{4}$ et La Vie des Fourmis, ${ }^{5}$ que du point de vue de l'importance primordiale et de la présence, à chaque instant, d'innombrables types de formes qui peuplent l'univers du vivant. L'auteur se sert de toute une série d'affirmations pour justifier l'existence d'une civilisation également au niveau des insectes ou des communautés végétales : il prouve de façon déductive que la présence d'une structure hiérarchisée ainsi qu'une organisation ayant un sens, démontrent la nécessité de formes de raison primaires qui gère ce genre de structures.

Il étudie avec la même précision les questions de la raison, c'est à dire de l'esprit de raison dans le domaine où il possède l'exclusivité, id est, chez l'homme. C'est ici que la psychométrie ${ }^{6}$ l'attire irrésistiblement : il l'exprime de façon concise dans la question : »Ayant éloigné les dieux et les morts, que reste-t-il «? ${ }^{7}$ Cette citation qui doit être comprise comme un artifice rhétorique, pourrait être prise pour des prolégomènes dans les nombreux écrits où il traite de l'eschatologie de la raison humaine, si l'on considère comme telle toute activité de l'esprit concrète et spéculative n'étant plus liée au corps qui lui assurait jadis le cadre matériel de son activité. Ceci ne peut prendre fin sans que la question de la conscience générale cosmique ne soit posée. C'est pourquoi il dit : »Nous voici donc devant le mystère de la conscience universelle. Bien que nous soyons incapables de comprendre l'acte d'un infini qui se replierait sur soi pour se sentir, par conséquent se définir et se séparer d'autre chose, ce n'est pas une raison suffisante pour le déclarer impossible: car si nous rejetions toutes les réalités et impossibilités que nous ne comprenons point, il ne nous resterait de quoi vivre «. ${ }^{8}$ Il tire une conclusion logique qu'il formule ainsi par la suite : »'il y a conscience et quelque lieu ou quelque chose qui remplace la conscience, nous serons dans cette chose, puisque nous ne pouvons être ailleurs. Et cette conscience ou cette chose ou nous nous trouverons, ne pouvant être malheureuse, puisqu'il est impossible que l'infini n'existe que pour son malheur, nous ne serons pas malheureux non plus «. ${ }^{9}$ Comme la mort reste la séparation permettant cela et rétablissant finalement tout, de même, la vie est impossible sans elle - »La vie, c'est la mort «, ${ }^{10}$ et que l'espace de l'infini qui s'étend derrière elle s'annonce dans le domaine du bonheur ou de l'éternel accomplissement ${ }^{11}$ et de ce fait, la pensée logique germant chez Maeterlinck est une pensée religieuse. En réalité, il a accordé une grande attention à la relation de l'Homme avec la transcendantalité des éléments essentiels de sa personnalité après la mort, comme en témoigne ses travaux : Le Grand Secret, ${ }^{12}$ La Sagesse et la Destinée, ${ }^{13}$ La Grande Loi

\footnotetext{
${ }^{2}$ Maeterlinck, Maurice. L'Intelligence des Fleurs. Paris : Charpentier, 1926.

${ }^{3}$ Maeterlinck, Maurice. La Vie des Abeilles. Paris : Charpentier, 1922.

${ }^{4}$ Maeterlinck, Maurice. La Vie des Termites. Paris : Charpentier, 1928.

${ }^{5}$ Maeterlinck, Maurice. La Vie des Fourmis. Paris : Charpentier, 1930.

${ }^{6}$ C'est l'expression que l'auteur utilise explicitement, même si elle a un sens différent dans la psychologie d'aujourd'hui.

${ }^{7}$ Maeterlinck, Maurice. L'Hôte inconnu. Paris : Charpentier, 1925 : 43.

${ }^{8}$ Maeterlinck, Maurice. La Mort, Paris : Charpentier et Fasquelle, 1929 : 267.

${ }^{9}$ Ibidem.

${ }^{10}$ Paul Chauchard. La Mort. Paris : Presses universitaires de France, 1966 : 6.

${ }^{11}$ Comparez la dernière phrase de l'affirmation dans la note no. 9.

${ }^{12}$ Maeterlinck, Maurice. Le Grand Secret. Paris : Charpentier-Fasquelle, 1925.

${ }^{13}$ Maeterlinck, Maurice. La Sagesse et la Destinée. Paris : Charpentier-Fasquelle, 1926.
} 
${ }^{14}$ et Avant le grand silence, ${ }^{15}$ si on ne s'arrête que sur les essentiels. Leur dénominateur commun pourrait encore être résumé en un traitement systématique du phénomène de l'homme (et de la conscience cosmique), dont la frontière ne se trouve pas sur la ligne reliant le berceau à la tombe, l'être avec le non-être, si l'on utilise l'une des images les plus courantes de l'auteur. ${ }^{16}$

C'est dans ce contexte que l'auteur croise la religion chrétienne. Pour commencer, il faut signaler que son rapport avec la grande religion révélée est contradictoire dans un sens aussi diachronique que synchronique. Ceci pourrait se rapporter notamment aux parties qui présentent une interprétation dogmatique des affirmations sans faire appel au mystère de l'initiation. Surtout si elles utilisent pour analogie directe la méthode reposant sur les descriptions anthropomorphiques. »Après tant d'efforts, après tant d'épreuves, nous nous retrouvons exactement au point d'où étaient partis nos grands instructeurs. Ils nous ont légué une sagesse que nous commençons a peine a débarrasser des débris que les siècles y avaient déposés; et sous ces débris nous retrouvons intact le plus haut aveu d'ignorance que l'homme ose proférer. C'est peu si l'on aime l'illusion, c'est beaucoup si l'on préfère la vérité. Nous savons enfin qu'il n'y eut jamais de révélation ultra-humaine, de message direct et irrécusable de la divinité, de secret ineffable et que tout ce que l'homme croit connaitre au sujet de Dieu, de don origine et de ses fins, c'est de sa propre raison qu'il l'a tiré«.. ${ }^{17}$ Cela est encore plus flagrant dans le chapitre $»$ Le Dieu d'Israël ${ }^{18}{ }^{18}$ qui fait partie des passages principaux du livre La Grande Porte. Il montre le bien fondé de la citation ci-dessus en demandant, avec une entière désillusion, pourquoi Jéhovah n'a pas agi lors de l'ultime blasphème que le roi Salomon lui avait préparé, quand ce dernier avait fait publiquement honorer le dieu Moabite tout en cédant à la polygamie. ${ }^{19}$ La réponse que propose Maeterlinck aurait un lien universel avec l'esprit d'un total agnosticisme, à savoir : Le dieu des Israéliens ne pouvait pas faire cela même s'il le voulait car ceci dépasserait ses forces. Il était inséparablement intégré dans le peuple qui avait imaginé son existence de même que tous ses attributs nécessaires à ce type de phénomène. Il n'était en réalité qu'un produit de leur pensée et de leur histoire culturelle. ${ }^{20}$ Donc, aucune révélation extrahumaine, venant vers un individu par un logos supérieur au monde, ne pouvait être possible. Grâce aux conclusions que l'on trouve dans d'autres passages parallèles du même chapitre, on peut logiquement en déduire que la seconde personne divine, qui est par nature égale au père, subit alors le même jugement.

Cependant Maurice Maeterlinck est un auteur chrétien. En plus du syllogisme qui montre par lui-même, qu'on ne peut pas éviter la question sur les religions monothéistes notamment sur la plus étendue parmi elles, au vu de l'œuvre dont les parties majoritaires sont consacrées à la réflexion sur les choses ultimes de la raison humaine

\footnotetext{
${ }^{14}$ Maeterlinck, Maurice. La Grande Loi. Paris : Charpentier-Fasquelle, 1930.

${ }^{15}$ Maeterlinck, Maurice. Avant le grand silence. Paris : Charpentier-Fasquelle, 1934.

${ }^{16}$ Ainsi: »Tout être étant inévitablement éternel, attend le non-être comme une délivrance impossible«, Maeterlinck, Maurice, La Grande Porte : 82.

${ }^{17}$ Maeterlinck, Maurice. Le Grand Secret : 316.

${ }^{18}$ Maurice Maeterlinck, La Grande Porte : $185-200$.

19 1R XI, 3.

${ }^{20}$ Comparez : Boštjan Marko Turk, »Maurice Maeterlinck et le dédale inextricable de l'existence «, Linguistica, 53/1, Ljubljana : Filozofska fakulteta, 2013. 251-263.
} 
et de sa conscience ; le nombre élevé de matériel cité par le poète et philosophe belge lors de ses travaux, plaide également en faveur de cette idée qui est liée à la Bible. Dans ce sens, une étude étendue est disponible, ${ }^{21}$ montrant de façon définitive à quel point la création de Maeterlinck est liée à l'univers de l'écriture révélée. Son ensemble présente l'idée que sans une inspiration christique, les éléments importants dans toute l'œuvre ne seraient pas ce qu'ils sont. C'est lorsque l'on prend en compte la lecture la plus approfondie de la Bible et la réflexion concernant ce thème actuel qu' on peut comprendre l'articulation théologique de l'affirmation suivante que l'on avance en contrepoint à la citation utilisée ci-dessus : Jésus répond aux docteurs: „En vérité, je Vous le dis, avant qu'Abraham ne fût, je suis«. Il ne dit pas j'étais. Il est depuis toujours, dans le passé comme dans le présent et dans l'avenir. Le Verbe n'a pas plus de temps puisqu'il est éternel. C'est l'un des rares moments où il parle comme parlerait notre Dieu. Il a ici le sens et le sentiment de son éternité «. ${ }^{22}$

Maeterlinck parle non seulement de façon affirmative de Dieu avec le pronom possessif de la première personne du pluriel, (et non du singulier, lui donnant de cette façon une forte valeur généralisatrice), mais il présente aussi l'union hypostatique qui unit les deux personnes divines en une entité, de la même façon que l'Église encore uniforme, à l'époque, le déterminait lors des conflits entre l'arianisme et ses formulations les plus extrêmes. ${ }^{23}$ Le présent éternel explique la divinisation humaine comme l'une des notions principales de la révélation, donnant ainsi à la littérature, s'alimentant de cette source, un sens particulier, qu'il est vain de chercher à la façon dont le traçait Maeterlinck en apparence dans ses premières citations, dans le domaine de l'agnosticisme intégral.

Par rapport à cela, on doit d'abord constater que l'existence intemporelle d'une union hypostatique n'est pas du tout en contradiction avec la condition humaine, qui tente de voir les créations spéculatives comme illusoires ou par conséquent, seulement comme un produit de sa propre imagination. Au contraire, le contenu du document de base de la chrétienté, c'est à dire de l'Ancien et du Nouveau Testament ne peut être compris si l'on ne consent pas à une telle contradiction à priori. Les textes du Nouveau et de l'Ancien Testament nous parlent d'ailleurs du drame de la vie humaine et de sa conscience qui pulse dans le temps, quantum ad statum viae pertinet, dans la multitude de formes qui sont déterminées par le scepticisme, la distance et le doute. Les auteurs inspirés des textes cités ont donné à l'humanité l'ambiguïté des concepts et des phénomènes avec lesquels l'homme est généralement confronté dans le domaine des sentiments, de la pensée et de l'existence en général. Ceci provoquait le tressaillement de la volonté créatrice sainte des auteurs entre reconnaissance et déni, où les œuvres dramatiques, surtout dans leur dimension tragique pénétraient dans chaque pore des textes inspirés par Dieu. Un exemple incomparable de cela reste les prophètes de l'Ancien Testament qui ont, avec un réalisme mimétique pour la condition humaine, placé en relation le chemin sur Terre avec lui-même et avec ses origines transcendantales.

\footnotetext{
${ }^{21}$ Lutaud, Christian. « Maeterlinck et la Bible«. Annales, 16. Bruxelles : Maurice Maeterlinck Stihting, $1971: 39-127$.

${ }^{22}$ Maeterlinck, Maurice. La Grande Porte, pp. 109-110.

${ }^{23}$ On trouve une excellente analyse de la citation ontologique chez : Kohlmann, Anthony. Unitarism philosophically and theologically examined. Washington : Guegan, 1821.
} 
Mais au début et à la fin de tout sentiment tragique se trouve une rupture cathartique. Elle apparait lorsque l'humain prend pleinement conscience de l'éloignement divin ainsi que de l'effrayant néant auquel il est remis déjà de son vivant ; il l'enveloppera totalement lors de la fin des temps, ce qui signifie pour chaque individu : le moment de sa mort. On trouve les textes les plus inspirants dans ce sens, dans la troisième partie de Tanaka, dans Les Ketouvim. ${ }^{24}$ C'est ici que ressort le problème de la théodicée, c'est à dire comment accorder le mal avec lequel on est confronté quotidiennement avec la relation entre Dieu et l'homme. L'éternel exemple de cette nouvelle contradiction est bien sûr l'histoire de Job, homme riche, empli de toute intégrité morale..$^{25}$ La Bible raconte qu'il est confronté au pire type d'expérience, lorsque son mode de vie récent s'effond ne laissant que la question du sens de la souffrance, de la mort et du rôle éventuel de Dieu dans cette dévastation. Les gens de son entourage qui lui disent de maudire Dieu et de mourir, amplifient le dilemme à un point insupportable, surtout car la première personne parmi eux est sa propre compagne. Il s'agit là d'une des unités à l'intonation la plus dramatique de la Bible, se terminant en une totale catharsis. L'homme n'est donc pas maître de son destin et ne peut l'influencer.

Cette notion marque d'autant plus l'histoire de la révélation avec le livre l'Ecclésiaste ${ }^{26}$ dans lequel le fils de David et roi d'Israël parle à son peuple. ${ }^{27}$ Son message disparaît progressivement dans un pessimisme concentré. Les exégètes et l'histoire biblique l'ont même désigné comme le passage de la révélation dans lequel Dieu est absent. ${ }^{28}$ On n'y trouve pas la plus petite épiphanie, seulement un appel tragico-absurde à la jouissance avant que tout ne s'éteigne. Au dessus des théorèmes laconiques avec lesquels le prophète plante le destin humain, se lève l'horizon d'un destin vide de sens et de la mort en tant que dernière frontière de tout ce qui vit. La mort est aussi le sens de la vie puisqu'elle rend plus heureux que la vie. »Etj'ai trouvé les morts qui sont déjà morts plus heureux que les vivants qui sont encore vivants «. ${ }^{29}$

Mais on peut comprendre que la Bible possède également d'autres éléments dans sa substance : En fait, il faut avouer que ce sont ces éléments qui représentent l'intentionnalité ultime, en vue de laquelle elle fut écrite. Les gens de l'entourage de Job, peuvent d'ailleurs êtres compris aussi grâce à l'image des guides aveugles et avant tout de la cécité qui a un fond spirituel exactement dans le sens de la formulation : »Laissezles, ce sont des aveugles qui guident des aveugles! Or si un aveugle guide un aveugle, tous les deux tomberont dans un trou«. ${ }^{30}$ La Bible s'exprime de façon encore plus sévère dans le cas des « autorités » majeures, qui sont d'autant plus grandes qu'elles attirent le plus grand nombre de personnes avec leurs analyses aveugles et, mieux encore, les guident vers une voie erronée. Ainsi : »Malheur à vous, guides aveugles, qui dites : «Si l'on jure par le sanctuaire, cela ne compte pas ; mais si l'on jure par l'or du sanctuaire, on est tenu. "Insensés et aveugles! Quel est donc le plus digne, l'or

\footnotetext{
${ }^{24}$ Nom original pour la dernière partie de la Bible hébraïque, Tanakh.

${ }^{25}$ Les Ketouvim, 16.

${ }^{26}$ Les Ketouvim, 20.

${ }^{27}$ Le sens étymologique du mot ecclésiaste est »celui qui parle à la foule«.

${ }^{28}$ Neher, André. Notes sur Quhélét. Paris : Les Éditions de Minuit, 1951.

${ }^{29}$ Ecc, 4, 2.

${ }^{30} \mathrm{Mt}, 15,14$.
} 
ou le sanctuaire qui a rendu cet or sacré«. ${ }^{31} \mathrm{~L}$ 'auteur des Ecclésiastes, pourrait alors être classifié dans cette catégorie sans pour autant remettre en question l'authenticité des livres canons. Si cela n'était pas ainsi, on inverserait alors complètement la multi dimensionnalité des expériences et pensées humaines. Dieu n'a pas éprouvé Job dans son dernier retranchement en lui enlevant encore sa vie, en plus de tout le reste. Mais ceci aurait pu également survenir et finit par arriver par un moyen naturel. Cependant, rien de tout cela n'ébranle en rien les notions essentielles de la foi qui sont centrés sur les miracles et notamment sur le plus grand d'entre-eux, c'est à dire, la résurrection. Les miracles sont la plus grande preuve de la puissance créatrice dans l'existence de l'homme, et ils ne peuvent briller que si on les place à côté de leur contrepoint, c'est à dire la mort, le néant, l'absence de sens et de Dieu. Ceci est l'intention dramatique et la fondation cathartique de la Bible ainsi, également, que de nos vies, dans la mesure où l'on se reconnaît dans ce domaine.

C'est aussi le contenu de la dramatique de Maeterlinck, dans laquelle il a établi la caractéristique principale de ce genre par le principe de la mise en abyme, ${ }^{32}$ à savoir que les événements et les phénomènes, les pensées et les sentiments, la vie et la mort des protagonistes ainsi que toutes les intrigues avec leurs projets et leurs «nonprojets », même si celles-ci demeurent inhabituelles pour la logique humaine, ne sont pas donnés de manière statique, de l'extérieur, à l'aide d'un narrateur omniscient, mais ils sont présentés en fonction de la dynamique interne de l'existence humaine et vus dans un moment synchrone d'un destin individuel et placés en tant que tels comme un exemple durable. La mise en abyme ou la mise en présence d'un événement en luimême est le fondement sur lequel est organisée la logique des textes de l'Ancien Testament et du Nouveau Testament. C'est à la façon d'un cône infinitésimal où les couches analogiques de contenu et de concept se transposent des parties plus étendues vers le sommet - par rapport à la question du sens et de la certitude, et surtout de la compréhension de la sphère humaine dans la transposition métaphysique - et se perdent dans le non-doute qui est notre plus grande certitude, quantum ad statum viae pertinet. ${ }^{33} \mathrm{Ceci}$ est le dénominateur commun de la texture dramatique entre la Bible et Maeterlinck qui, en fonction du type, ne peut s'appliquer qu'à celle dont il est question ici.

Pour déterminer de façon plus précise l'orientation de la présente réflexion, on cite alors l'impulsion diachronique qui est un élément essentiel de la structure chrétienne du monde, un espoir basé sur la paix et la certitude dans la foi, même si toutes les coordonnées d'une telle activité ne sont pas claires. Elle aide à déterminer quand l'élan vers le christianisme est le plus intense chez Maurice Maeterlinck, ainsi ipso facto, en fonction de la coïncidence temporelle, de la formation des œuvres dramatiques les plus importantes, elles montrent comment le sentiment d'inquiétude cédait place à celui

\footnotetext{
${ }^{31} \mathrm{Mt}, 23,16-17$.

${ }^{32}$ Maeterlinck connaissait bien ce procédé issu de la peinture flamande : „Encore une fois, un immense problème se trouve effleuré en une phrase. Maeterlinck avait trop bien regardé les tableaux des maîtres flamands pour n'avoir pas observé cette présence du miroir »en abyme«. Et il dégage (...) la question de la symbolique du miroir, en faisant déboucher ce problème de technique, picturale ou littéraire, dans sa préoccupation personnelle, qui est celle de ses contemporain: comment présenter le mystère sans le trahir? Comment suggérer l'invisible? « Pouillard, Raymond. »Un projet et quelques lectures«, Annales, 10, Bruxelles, Maurice Maeterlinck Stichting, $1964: 13$.

${ }^{33}$ Voir supra.
} 
de la paix et de la tranquillité. Il est un fait que les drames les plus importants chez Maeterlinck tombent juste à la période déterminée par la citation suivante : $\gg$ Comme toute l'œuvre d'avant 1895 est sous le signe de l'inquiétude, toute celle d'après 1889, d'après Sagesse et Destinée qui est un livre non de paix, mais d'apaisement et ou l'on enregistre les dernières vibrations de l'inquiétude maeterlinckienne - cette œuvre, disje, qu'inaugure La Vie des Abeilles, n'est elle pas sous le signe de la tranquillité? Partie du soupir, de l'exclamation, du monosyllabe, presque du hoquet avec la Princesse Maleine, la phrase maeterlinkcienne devient l'une des plus larges, des plus nombreuses, des plus majestueusement simples qui aient jamais été écrite dans notre langue. Cette phrase, en fait, devient si ample et si égale qu'elle cesse d'être une phrase dramatique, comme il apparaît dans Marie-Magdeleine, pièce superbe, qui devrait émouvoir, et qui n'émeut que médiocrement. Que de critiques ce Silanus n'a pas irrités, avec sa sérénité que ne trouble pas même la vue d'un mort qu'on ressuscite! Pourtant Silanus ressemble a Maeterlinck. C'est Maeterlinck tranquillisé, voila tout«. ${ }^{34}$

Tous les drames principaux ayant pour thème la chrétienté, correspondent à cette période. Une légère exception serait Les Aveugles ${ }^{35}$ dont la première édition est sortie en 1890, mais son thème biblique est tellement évident qu'il se classe dans cette catégorie en fonction de l'inspiration générale. Indubitablement se trouve aussi dans cette catégorie et chronologiquement : Soeur Beatrice (1901), ${ }^{36}$ L'Oiseau bleu (1909), ${ }^{37}$ Marie-Magdeleine (1913) ${ }^{38}$ ainsi que Le Miracle de Saint Antoine (1920). ${ }^{39}$ Les plus intenses parmi eux déterminent même un phénomène de stationnarité transcendantale de la langue, qui perd ainsi sa fonction de message artistique pour se concentrer sur les secrets ontologiques du vivant. Comme le dit la phrase en exergue, la langue poétique se brise pour ouvrir une voie au signifié ineffable.

Observons l'œuvre qui puise ici, de la façon la plus large possible, MarieMagdeleine..$^{40}$ Pour mieux le comprendre il faut à nouveau penser à l'affirmation qui sert de point de départ et dont on s'est servi pour expliquer l'orientation essentielle de la voie créative de l'artiste, ce qui est une relation entre l'existence intemporelle de la conscience humaine et sa mise en place dans l'infini. Ainsi : »S'il y a conscience et quelque lieu ou quelque chose qui remplace la conscience, nous serons dans cette chose, puisque nous ne pouvons être ailleurs. Et cette conscience ou cette chose ou nous nous trouverons, ne pouvant être malheureuse, puisqu'il est impossible que l'infini n'existe que pour son malheur, nous ne serons pas malheureux non plus. ${ }^{41}$ On ne peut pas comprendre cette citation si l'on ne la relie pas avec les phrases dans lesquelles Maeterlinck s'est plongé dans la matrice évangélique avec tout ce dont son énergie hu-

\footnotetext{
${ }^{34}$ Doneck, Guy. Maurice Maeterlinck - une poésie - une sagesse - un homme, Bruxelles : Palais des Académies, $1961: 237$.

${ }^{35}$ Maeterlinck, Maurice. Les Aveugles, Paris : Charpentier, 1925. (On cite les éditions selon lesquelles les citations sont résumées dans l'article, elle contiennent aussi l'année de la première parution de chaque œuvre dramatique en particulier).

${ }^{36}$ Maeterlinck, Maurice. Soeur Beatrice. Paris : Charpentier, 1925.

${ }^{37}$ Maeterlinck, Maurice. L'Oiseau bleu. Paris : Fasquelle, 1956.

${ }^{38}$ Maeterlinck, Maurice. Marie-Magdeleine. Paris : Charpentier, 1922.

${ }^{39}$ Maeterlinck, Maurice. Les Aveugles. Paris : Charpentier, 1925.

${ }^{40}$ Voir supra.

${ }^{41}$ Voir supra.
} 
maine et artistique était capable, tant il a enrichi le verset biblique. ${ }^{42}$ Citons à nouveau : Jésus répond aux docteurs: "En vérité, je Vous le dis, avant qu'Abraham ne fût, je suis«. Il ne dit pas j'étais. Il est depuis toujours, dans le passé comme dans le présent et dans l'avenir. Le Verbe n'a pas plus de temps puisqu'il est éternel. C'est l'un des rares moments où il parle comme parlerait notre Dieu. Il a ici le sens et le sentiment de son éternité« ${ }^{43} \mathrm{Si}$, à la lumière, de ces mots, on se tourne vers le phénomène dans lequel on pourrait confronter en un dénominateur commun indispensable les textes cités, nous n'aurons qu'une solution à notre disposition. C'est le miracle, lorsque l'homme vainc la mort, lorsqu'il brise le malheur et le mal, et s'établit dans l'éternité de cet état que nous nommons de façon moins claire, bonheur, et en termes plus techniques on transmet son essence dans des approximations analogiques : « résurrection d'entre les morts », «le salut », « l'éternité » ou «l'infini ». Le bonheur intemporel de la conscience humaine dans l'éternité, présupposée par la résurrection, englobe l'intemporalité du Christ, comme il est écrit dans la Bible et l'on peut la résumer en une synthèse. Dans ce sens c'est aussi la seule réponse possible à la problématique complexe de la mort, comme on vient de la présenter en introduction et qui occupe intégralement la curiosité artistique et humaine du dramaturge belge.

L'œuvre prédominante dans ce sens est, comme constaté, Marie-Magdeleine. Son contenu peut être déverrouillé en utilisant la procédure qu'on a déjà définie chez Maeterlinck, la mise en abyme. L'œuvre contient deux points centraux. Le premier est la mort et la résurrection de Lazar, le second est quant à lui la mort du Christ et sa résurrection. Le premier se retrouve dans le second, c'est son image miroir. Tel Lazar qui mourut une fois et fut ramené à la vie, selon les termes de la Bible nous prendrons tous part à cela, aux derniers jours. à ce moment là, le Christ sera «tout en tous $»^{44} \mathrm{car}$ la vie sera séparée de la mort - c'est cette vie qu'il a légué à l'espèce homo sapiens sapiens, avec l'événement par lequel se conclut Marie-Magdeleine. Dans la résurrection de Lazar on voit le secret caché de la résurrection du Christ et par conséquent celui de notre destin eschatologique, ce qui est l'essence de la technique de mise en abyme qui attirait Maurice Maeterlinck. Citons le paragraphe le plus caractéristique : »Le cadavre était la, sous la lumière avide qui dévorait la grotte, couché comme une statue informe, rigide, étroite, serrée de bandelettes, le visage couvert d'un suaire. La foule, tassée en demi-cercle, irrésistiblement attiré et repoussée, se penchait, tendait ses mille cous, sans oser approcher. Le Nazaréen se tenait seul, en avant. Il leva la main, dit quelques

\footnotetext{
${ }^{42}$ Ces réflexions coïncident avec les éléments relatifs à ce que donne la critique littéraire ou l'histoire. L'âme humaine devrait s'unir au Christ hors du temps dans une sorte de présent éternel. Ainsi : »En revanche, chez certains qui sont les avertis, une liaison plus ou moins habituelle existerait entre la conscience claire et cette conscience profonde qui sait les choses de l'avenir puisque l'âme, principe permanent, est de demain comme d'hier. On voit se profiler a l'horizon d'une telle théorie le postulat de la préexistence de l'avenir que plus tard le philosophe cherchera a préciser dans le concept de l'éternel présent«, Vivier, Robert. »Histoire d'une âme «. Maurice Maeterlinck, 1862 - 1962. Bruxelles : La Renaissance du livre, 1962 : 163. D'autres auteurs voient les choses de façon similaire. Ainsi : »Materlinck considère (et peut être) faut il voir en ceci un réflexe de défense, (ou une consolation), il considère, dans ses essais, que le temps n'existe point de façon absolue, qu'il est réversible, que tout un événement futur existe déjà, aussi réel qu'un événement passé«, Compère, Gaston. Le Théâtre de Maurice Maeterlinck. Bruxelles : Palais des Académies, 1955 : 249. Pour »l'éternel présent » comparez aussi Turk, Boštjan Marko. Paul Claudel et l'actualité de l'être. Paris : Téqui, 2011.

${ }^{43}$ Voir supra.

${ }^{44}$ Eph, 4, 6.
} 
mots que je ne saisis point, puis d'une voix dont je n'oublierai jamais la puissance captive, s'adressant au cadavre, il s'écria: »Lazare, sors! $\ll .{ }^{45}$ Lazar se lève et marche dans la vie. La rigidité de la langue qui se libère de sa fonction directe pour se refléter dans la réalité, hors du réel, de la vie en Dieu »par lui-même » ne doit pas leurrer pour empêcher de voir en ce texte un des témoignages les plus forts de la détermination eschatologique humaine. Sans tenir compte de l'affirmation de Gaston Compere, ${ }^{46}$ où il critique le calme de Silanus lors de l'événement qui dépassait la connaissance et la compréhension de chacune des personnes présentes, on doit, dans la dignité stoïque de celui-ci, voir le plus grand témoignage de la véracité de l'événement, et surtout de sa signification, dans le sens générique de l'espèce de l'homme.

À la lumière de ceci, on peut aussi se tourner vers le nombre des détails particulièrement significatifs de la fin du drame. C'est là que Marie-Magdeleine rencontre le Christ qui part vers l'éternité. Cependant, cette rencontre est de nouveau présente à travers une nouvelle mise en abyme. On la voit notamment à travers le prisme du héros d'un des principaux drames chrétiens de l'auteur belge, c'est à dire par le prisme de l'homme aveugle. Citons :»L'Aveugle de Jéricho, a la fenêtre: »Il tombe!... Il est tombé!... Il regarde la maison!.... (...) L'Aveugle de Jéricho, à la fenêtre Il se relève!... Ils l'entrainaient!... Le tumulte, les cris de mort reprennent et redoublent dans la rue. Verus sort lentement en regardant Marie-Magdeleine qui est demeurée immobile, comme en extase, et tout illuminée de la clarté des torches qui s'éloignent «. ${ }^{47}$

L'homme aveugle qui est le fondement du drame chrétien, Les Aveugles dans les œuvres de Maurice Maeterlinck, témoigne en faveur de la lumière. La parabole de Job dans l'Ancien Testament qui - en absent - confirme Dieu, ne pourrait pas être mieux placée. Les aveugles voient et guident. à la lumière de cette maxime, on pourrait aussi se tourner vers les œuvres suivantes du cycle dramatique de Maeterlinck et notamment vers Sceur Beatrice ${ }^{48}$ ainsi que Le Miracle de saint Antoine ${ }^{49}$ La même logique se confirme également ici. Le spectre dramatique de Maurice Maeterlinck, comme il s'est façonné dans sa période mûre, ${ }^{50}$ ne pourrait être compris sans prendre en compte le contact intégral que l'auteur avait avec la Bible et l'héritage chrétien. Il n'est d'ailleurs possible de comprendre Sour Béatrice également que dans ce contexte, puisqu'il est sous titré de façon formelle «Le miracle ». Le miracle est entièrement en rapport avec l'image du Job de l'Ancien Testament et de son histoire: quand il pense qu'il est le plus loin de Dieu, c'est alors qu'il en est le plus près. L'histoire parle de l'échange d'identité entre la vierge Marie et la religieuse éponyme de l'Abbaye de Gand au 15ème siècle. Bien que sœur Béatrice se juge comme la dernière parmi les adoratrices de la Sainte Mère à cause des pêchés de désirs corporel et de non chasteté, cette dernière la place alors en premier : elle prend son identité et est prête à supporter la punition qui lui est dévolue. L'histoire littéraire s'est déjà prononcée la-dessus: »The Abbesse enters, followed by two nuns. They notice the missing statue and accuse »Sister Beatrice of allowing its theft. She does not deny the charge and she is condemned by the Abess to

\footnotetext{
${ }^{45}$ Maeterlinck, Maurice. Marie-Magdeleine : 86-87.

${ }^{46}$ Voir supra.

${ }^{47}$ Maeterlinck, Maurice. Marie-Magdeleine : 180.

${ }^{48}$ Maeterlinck, Maurice., Théâtre, 3. Paris : Charpentier, 1925.

${ }^{49}$ Maeterlinck, Maurice. Théâtre, 2. Paris : Charpentier, 1925.

${ }^{50}$ Voir supra, la citation de Gaston Compère.
} 
be whipped. Even so, she will not affirm or deny how the image disappeared $«{ }^{51}$ Le fait que ces deux personnes, celle qui a donné au monde un sauveur et la nonne flamande anonyme, soient égalisées aux yeux des sciences littéraires, est énoncé par les guillemets entourant le prénom de l'une d'elles, dans la citation littérale ci-dessus. ${ }^{52}$ Ainsi, la conclusion présente est placée sur un fondement objectif. Il est nécessaire seulement de conclure que la réalisation dramatique de Maeterlinck s'appuie ici aussi sur un miroir caché, une mise en abyme, qui nous permet de voir les relations interpersonnelles dans la perspective de transcendance du destin de l'homme.

Il existe aussi une autre conversion de l'histoire de Job, se déroulant dans le sens contraire de celles citées jusque là, en somme, que l'homme est très loin de Dieu lorsqu'il croit en être le plus près. Sur ce point, on ne peut repousser l'analogie qui est rétablie par les extraits citées de L'Ecclesiaste et du Miracle de Saint Antoine. ${ }^{53}$ Dans le monde petit bourgeois qui se caractérise par sa suffisance, arrive Saint Antoine. Son but est universel. Selon le modèle biblique, il veut ressusciter Mademoiselle Hortense, morte à la 77ème année de sa vie, après un repos de trois à quatre jours dans la tombe. Il dit : »Je veux ressusciter Mademoiselle Votre Tante « ${ }^{54}$ et il ajoute: »Il faut que je la ressuscite maintenant $\ll .{ }^{55}$ Mais il se confronte alors à une totale incompréhension : il est finalement même chassé des lieux en tant que dément et ivrogne. Il ramène néanmoins Mademoiselle la tante à la vie, mais elle retombe bien vite à nouveau dans le sommeil éternel : sans comprendre l'action du saint, et encore moins de pouvoir l'apprécier. Étroit est le chemin qui mène vers la vie, nombreux sont ceux qui y marchent mais ils ne le voient pas : Mais souvent le voient justement que ceux qui, aveugles, détectent la lumière de la vie de l'au-delà, comme le disent les exemples décrits, de fait ou par analogie. Mais que cela reste un travail à exécuter une prochaine fois : »Sunt autem et alia multa quae fecit Iesus: quae si scribantur per singula, nec ipusm arbitror mundum capere eos qui scribendi sunt libros «. ${ }^{56}$ On trouve beaucoup de ce genre de choses, sous cette forme ou sous une autre, justement dans l'œuvre de l'auteur flamand auquel nous avons consacré le document présent ; le mot poétique, surtout dans les drames, se base sur ce qui ouvre le chemin menant à la réalité qui se cache encore dans le pénombre, comme dit in exergo.

Université de Ljubljana, Slovénie

\footnotetext{
${ }^{51}$ Mahony, Patrick. Maurice Maeterlinck, mystic and dramatist. Washington: The Institute for the study of man, 1984: 95.

${ }^{52}$ Id est: »Sister Beatrice«.

${ }^{53}$ Cité supra.

${ }^{54}$ Maeterlinck, Maurice. œeuvres II. Bruxelles : Éditions Complexes, 1999 : 414.

${ }^{55}$ Ibidem.

${ }^{56} \mathrm{Jn}, 25$.
} 


\section{BIBLIOGRAPHIE :}

Chauchard, Paul. La Mort. Paris : Presses universitaires de France, 1966.

Chinatti, Claudia. »Fausto Maria Martini, poète crépusculaire italien, disciple de MaeteMaurice«. Maurice Maeterlinck Stichting, 1965. 7-31.

Compère, Gaston. Le Théâtre de Maurice Maeterlinck. Bruxelles: Palais des Académies, 1955.

Doneck, Guy. Maurice Maeterlinck - une poésie - une sagesse - un homme. Bruxelles: Palais des Académies, 1961.

Kohlmann, Anthony. Unitarism philosophically and theologically examined, Washington: Guegan, 1821.

Lutaud, Christian. »Maeterlinck et la Bible«. Annales, 14. Bruxelles: Maurice Maeterlinck Stihting, 1971. 39-127.

Maeterlinck, Maurice. Avant le grand silence. Paris: Charpentier, 1934.

Maeterlinck, Maurice. Les Aveugles. Paris : Charpentier, 1925.

Maeterlinck, Maurice. Le Grand Secret. Paris : Charpentier, 1925.

Maeterlinck, Maurice. La Grande Loi. Paris: Charpentier, 1930.

Maeterlinck, Maurice. La Grande Porte. Paris : Charpentier, 1939.

Maeterlinck, Maurice. L'Hôte inconnu. Paris : Charpentier, 1925.

Maeterlinck, Maurice. L'Intelligence des Fleurs. Paris : Charpentier, 1926.

Maeterlinck, Maurice. Marie Magdeleine. Paris : Charpentier, 1922.

Maeterlinck, Maurice. La Mort. Paris : Charpentier, 1929.

Maeterlinck, Maurice. ouvres II. Bruxelles : Éditions Complexes, 1999.

Maeterlinck, Maurice. L'Oiseau bleu. Paris : Fasquelle, 1956.

Maeterlinck, Maurice. La Sagesse et la Destinée. Paris : Charpentier, 1926.

Maeterlinck, Maurice. Théâtre II. Paris : Charpentie, 1923.

Maeterlinck, Maurice. Théâtre III. Paris : Charpentier, 1925.

Maeterlinck, Maurice. La Vie des Abeilles. Paris : Charpentier, 1922.

Maeterlinck, Maurice. La Vie des Fourmis. Paris: Charpentier, 1930.

Maeterlinck, Maurice. La Vie des Termites. Paris : Charpentier, 1928.

Mahony, Patrick. Maurice Maeterlinck, mystic and dramatist. Washington, D.C.: The Institute for the study of man, 1984.

Neher, André. Notes sur Quhélét. Paris: Les Éditions de Minuit, 1951.

Pouillard, Raymond. »Un projet et quelques lectures«. Annales, 10, Bruxelles: Maurice Maeterlinck Stichting, 1964. 7-20.

Turk, Boštjan Marko. »Maurice Maeterlinck et le dédale inextricable de l'existence«. Linguistica, 53/1. Ljubljana: Filozofska fakulteta, 2013. 251-263.

Vivier, Robert. »Histoire d'une ame«. Maurice Maeterlinck, 1862 - 1962. Bruxelles: La Renaissance du livre, 1962. 